\title{
Noncommutative Symmetric Hall-Littlewood Polynomials
}

\author{
Lenny Tevlin
}

New York University, New York, NY, USA

\begin{abstract}
Noncommutative symmetric functions have many properties analogous to those of classical (commutative) symmetric functions. For instance, ribbon Schur functions (analogs of the classical Schur basis) expand positively in noncommutative monomial basis. More of the classical properties extend to noncommutative setting as I will demonstrate introducing a new family of noncommutative symmetric functions, depending on one parameter. It seems to be an appropriate noncommutative analog of the Hall-Littlewood polynomials.

Résumé. Les fonctions symétriques non commutatives ont de nombreuses propriétés analogues à celles des fonctions symétriques classiques (commutatives). Par exemple, les fonctions de Schur en rubans (analogues de la base de Schur classique) admettent des développements à coefficients positifs dans la base des monômes non commutatifs. La plupart des propriétés classiques s'étendent au cas non commutatif, comme je le montrerai en introduisant une nouvelle famille de fonctions symétriques non commutatives, dépendant d'un paramètre. Cette famille semble être un analogue non commutatif approprié de la famille des polynômes de Hall-Littlewood.
\end{abstract}

Keywords: symmetric functions

\section{Introduction and Results.}

Classical (commutative) Hall-Littlewood polynomials are well studied objects with applications ranging from representation theory Desarmenien et al. (1994) to Bethe ansatz in math physics Kirillov and Reshetikhin (1988). While there have been several attempts to find an object in the algebra on noncommutative symmetric function that would mimic some of the properties of the classical Hall-Littlewood polynomials Hivert (1998); Bergeron and Zabrocki (2005); Novelli et al. (2010) each with its own merit, I think it is worthwhile to continue the search. In particular, the new noncommutative symmetric functions I will introduce in 5 enjoy the following properties

- they reduce to noncommutative ribbon Schur functions at $t=0$

- they reduce to noncommutative monomial functions at $t=1$

- coefficients of the expansion of ribbon Schur functions in the new basis are polynomials in $t$ with positive integer coefficients;

1365-8050 @ 2011 Discrete Mathematics and Theoretical Computer Science (DMTCS), Nancy, France 


\section{Operations on Compositions}

To set the stage, I need some definitions, most of which are standard. Bases of the algebra of noncommutative symmetric functions are labeled by compositions, which will be denoted by capital Latin letters.

Let $I=\left(i_{1}, \ldots, i_{n}\right)$ be a composition, i.e. an ordered set of positive integers $\left(i_{1}, \ldots, i_{n}\right)$, called parts of the composition $I$. The sum of all parts of a composition, its weight, is denoted by $|I|$ and the number of parts in the composition, its length - by $\ell(I)$. Alternatively, given the weight of a composition $I$, it can be specified by its descent set, $D(I)$. If $D(I)=\left\{d_{1}, \ldots, d_{k}\right\}$ with $d_{1}<d_{2}<\ldots<d_{r}<|I|-1$, then $I=\left(d_{1}, d_{2}-d_{1}, \ldots,|I|-d_{k}\right)$. A descent set can be defined for any word from an ordered alphabet as the set of positions of letters, which are greater than their right neighbor.

Major index of a composition with $n$ parts is $\operatorname{maj}(I)=\sum_{k}(n-k) i_{k} \equiv \sum_{r} d_{r}$.

Two types of multiplication for compositions were defined in Gelfand et al. (1995). For two compositions $I=\left(i_{1}, \ldots, i_{r-1}, i_{r}\right)$ and $J=\left(j_{1}, j_{2}, \ldots, j_{s}\right)$ define

$$
\begin{aligned}
& I \triangleright J=\left(i_{1}, \ldots, i_{r-1}, i_{r}+j_{1}, j_{2}, \ldots, j_{s}\right), \\
& I \cdot J=\left(i_{1}, \ldots, i_{r}, j_{1}, \ldots, j_{s}\right),
\end{aligned}
$$

Reverse refinement order for compositions is defined as follows. Let $I=\left(i_{1}, \ldots, i_{n}\right), J=\left(j_{1}, \ldots, j_{s}\right)$, $|J|=|I|$. Then $J \preceq I$ if every part of $J$ can be obtained from consecutive parts of $I$ :

$$
J=\left(i_{1}+\ldots+i_{p_{1}}, i_{p_{1}+1}+\ldots+i_{p_{2}}, \ldots, i_{p_{k-1}+1}+\ldots+i_{p_{k}}, \ldots, i_{p_{s}}+\ldots+i_{n}\right)
$$

for some nonnegative $p_{1}, \ldots, p_{s}$. (The convention $p_{0}=0$ will be implied below.) The composition $\left(p_{1}, \ldots, p_{s}\right)$ will be denoted by $I_{J}$.

Example 1 For instance, consider $I=(3,3,2)$ :

$$
|(3,3,2)|=8 \quad \ell(3,3,2)=3 \quad D(3,3,2)=\{3,6\}
$$

Also $(3,3,2)=(3,1+2,2) \preceq(3,1,2,2)$.
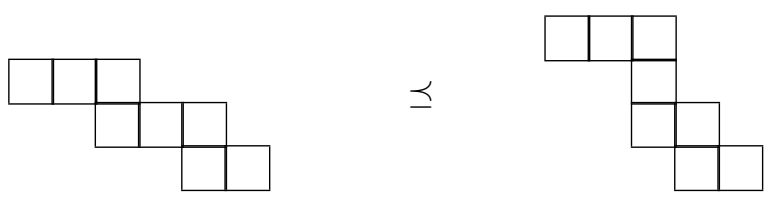

Here $p_{1}=1, p_{2}=3, p_{3}=4$, i.e. $I_{J}=(1,3,4)$

\section{Standard Bases for Noncommutative Symmetric Functions}

The algebra NSym, introduced originally in Gelfand et al.(1995), is a noncommutative associative graded algebra generated by noncommutative power sums (of the first kind) $\Psi_{k}$ of degree $k$. Products of power sums form a multiplicative basis:

$$
\Psi^{I}=\Psi_{i_{1}} \cdot \ldots \cdot \Psi_{i_{k}}
$$

As in the classical (commutative) theory of symmetric functions, there is a number of useful bases of NSym. Noncommutative monomial symmetric functions Tevlin (2007) can be defined as 
Definition 1

$$
M^{I}=\sum_{J \preceq I} \frac{(-1)^{\ell(I)-\ell(J)}}{\prod_{k=0}^{s-1}\left(\ell(I)-p_{k}\right)} \Psi^{J}
$$

where $s=\ell(J)$.

Example 2 Suppose the task is to obtain $M^{312}$. There are four compositions $J$, such that $J \preceq 312$ :

$$
\begin{array}{lllll}
312 & \text { with } & p_{1}=1 & p_{2}=2 & p_{3}=3 \\
3^{2} & \text { with } & p_{1}=1 & p_{2}=3 \\
42 & \text { with } & p_{1}=2 & p_{2}=3 \\
6 & \text { with } & p_{1}=3 & &
\end{array}
$$

Therefore

$$
M^{312}=\frac{1}{3}\left(\Psi_{6}-\Psi_{4} \Psi_{2}-\frac{1}{2} \Psi_{3}^{2}+\frac{1}{2} \Psi_{3} \Psi_{1} \Psi_{2}\right)
$$

Noncommutative complete (homogeneous) symmetric function $S_{n}$ can be defined as

Definition 2

$$
S_{n}=\sum_{|I|=n} M^{I}
$$

with a corresponding multiplicative basis functions

$$
S^{I}=\prod_{k=1}^{\ell(I)} S_{i_{k}}
$$

Finally, noncommutative ribbon Schur basis has been defined by Gelfand et al. (1995) as:

Definition 3

$$
R^{I}=\sum_{J \preceq I}(-1)^{\ell(I)-\ell(J)} S^{J}
$$

\section{Transition between Different Bases.}

\subsection{Ribbon and Monomial.}

Analogously to the situation on the commutative theory, where Schur functions expand positively in the monomial basis, noncommutative ribbon Schur functions expand in the noncommutative monomial basis with positive coefficients. This was proved in Hivert et al. (2009), who also provided the combinatorial meaning of coefficients.

Proposition 1 (Hivert et al. (2009)) The coefficient $K_{I J}$ in the expansion

$$
R^{I}=\sum_{J} K_{I J} M^{J}
$$

is equal to the number of packed words such that the descent set of $u, D(u)=I$ and the word count $W C(u)=J$.

In the following section I recall definitions of objects in this statement as the main results of the present paper generalize this result. 


\subsection{Compositions Associated to Packed Words.}

Packed words are defined the following way:

Definition 4 The packed word $u=\operatorname{pack}(w)$ associated with a word $w \in A$ ( $A$ is an alphabet) is obtained by the following process. If $b_{1}<b_{2}<\ldots<b_{r}$ are the letters occurring in $w, u$ is the image of $w$ by the homomorphism $b_{i} \mapsto a_{i}$. A word $u$ is said to be packed if pack $(u)=u$.

Example 3 Restricting A to the first five integers, the following is the list of words $w_{k}$, such that pack $\left(w_{k}\right)=$ 13132:

$$
\begin{aligned}
& w_{1}=13132, w_{2}=14142, w_{3}=14143, w_{4}=24243, w_{5}=15152, w_{6}=15153 \\
& w_{7}=25253, w_{8}=15154, w_{9}=25254, w_{10}=35354
\end{aligned}
$$

Put differently, these are words in which if each letter is replaced by a smaller one (respecting the order $1<2<\ldots$ ), one gets the word made out of smallest possible letters, $u=13132$. A packed word is such that cannot be simplified this way.

There are two ways to associate a composition to each packed word $u$ :

1. First one considers a descent set of a word, $D(u)$, and builds a corresponding composition. For instance, if $u=2113$, its the descent set $\{1\}$. Therefore the corresponding composition is (13); for $u=3221, D(3221)=\{1,3\}$, which corresponds to $(121)$.

2. Secondly, the word composition $W C(u)$ of $u$ is the composition whose descent set is given by the positions of the last occurrences of each letter in $u$. For example,

$$
W C(1543421323)=(23221)
$$

Indeed, the descent set is $\{2,5,7,9,10\}$ since the last 5 is in position 2 , the last 4 is in position 5 , the last 1 is in position 7, the last 2 is in position 9 , the last 3 is in position 10.

Finally, with each word one can associate a statistics $\operatorname{sinv}(u)$, called special inversion, that counts the number of times the last occurrence (reading from left to right) of a smaller letter is to right of a larger one Novelli et al. (2010). For example,

$$
\operatorname{sinv}(3221)=4
$$

since the second 2 is to the right of 3,1 is to right of both 2 's and 3 .

\subsection{Monomial and Complete.}

In this section I will find the explicit formula for the coefficients $\varrho_{J \mid K}$ in the following expansion

$$
M^{J}=\sum_{K} \varrho_{J \mid I} S^{I}
$$


Consider two compositions $I$ and $J$ and take the first (largest) $K$ such that $K \preceq I$ and $K \preceq J$. Put differently, $D(K)=D(I) \cap D(J)$. More precisely, let

$$
\begin{aligned}
& I=\left(i_{1}, \ldots, i_{n}\right) \\
& J=\left(j_{1}, \ldots, j_{l}\right) \\
& K=\left(k_{1}, \ldots, k_{m}\right) \quad \text { with } \quad m \leq n, \quad m \leq s \\
& K=\left(i_{1}+\ldots+i_{p_{1}}, \ldots, i_{p_{m-1}+1}+\ldots+i_{n}\right) \\
& K=\left(j_{1}+\ldots+j_{r_{1}}, \ldots, j_{r_{m-1}+1}+\ldots+j_{l}\right)
\end{aligned}
$$

\section{Proposition 2}

$$
\varrho_{J \mid I}=(-1)^{\ell(I)-\ell(J)} \frac{\prod_{s \in\{1, \ell(J)\} /\left\{r_{k}\right\}}(\ell(J)-s) \prod_{k=1}^{m}\left(\ell(J)-r_{k}+i_{p_{k}}\right)}{\ell(J) !}
$$

Proof (sketch): The expansion of power sums in complete is known Gelfand et al. (1995). Inserting this expansion in the definition of monomials above one gets a fairly ugly sum, which can be recognized as an expansion of a certain determinant. Calculation of the determinant results in (3).

Example 4 Consider $\varsigma_{21^{2} \mid 1^{2} 2}$

$$
\begin{aligned}
& K=2^{2} \quad \ell(K) \equiv m=2 \\
& I=21^{2} \Rightarrow \begin{cases}p_{1}=1 & i_{p_{1}} \equiv i_{1}=2 \\
p_{2}=3 & i_{p_{2}} \equiv i_{3}=1\end{cases} \\
& J=1^{2} 2 \Rightarrow\left\{\begin{array}{l}
r_{1}=2 \\
r_{2}=3
\end{array}\right. \\
& \{1,2,3\} /\{2,3\}=1
\end{aligned}
$$




\section{Noncommutative Hall-Littlewood Polynomials.}

This section contains one the most important results of this paper. Below I will define what seems to be an appropriate noncommutative analog of Hall-Littlewood Polynomials. Classical Hall-Littlewood polynomials $P_{\boldsymbol{\lambda}}(t)$ enjoy the following properties Macdonald (1995) (please refer to this book for notations):

1.

$$
P_{\boldsymbol{\lambda}}(0)=s_{\boldsymbol{\lambda}}
$$

2.

$$
P_{\boldsymbol{\lambda}}(1)=m_{\boldsymbol{\lambda}}
$$

3.

$$
s_{\boldsymbol{\lambda}}=\sum_{\boldsymbol{\kappa}} K_{\boldsymbol{\lambda} \boldsymbol{\kappa}}(t) P_{\boldsymbol{\kappa}}(t),
$$

where Kostka-Foulkes polynomials $K_{\lambda \kappa}(t)$ are polynomials in $t$ with positive integer coefficients, whose combinatorial meaning is well-understood Lascoux and Schutzenberger (1978), Kirillov (1998).

Polynomials $P^{I}(t)$ to be introduced below have the following properties

1.

$$
P^{I}(0)=R^{I}
$$

2.

$$
P^{I}(1)=M^{I}
$$

3.

$$
R^{I}=\sum_{J} K_{I J}(t) P^{J}(t),
$$

where $K_{I J}(t)$ are polynomials in $t$ with positive integer coefficients, whose meaning will be explaned below.

4. Moreover in the commutative limit noncommutative Hall-Littlewood polynomials for hook compositions $I=n 1^{k}$ reduce to classical Hall-Littlewood polynomials $Q_{\boldsymbol{\lambda}}(t)$ where $\boldsymbol{\lambda}=n 1^{k}$.

I will define noncommutative Hall-Littlwood polynomials through their expansion in complete basis. Once again for two compositions $I$ and $J$ select the first (largest) $K \preceq I, K \preceq J$ (i.e. $D(K)=D(I) \cap$ $D(J)$ ) and let

$$
\begin{aligned}
& I=\left(i_{1}, \ldots, i_{n}\right) \\
& J=\left(j_{1}, \ldots, j_{s}\right) \\
& K=\left(k_{1}, \ldots, k_{m}\right) \quad \text { with } \quad m \leq n, \quad m \leq s \\
& K=\left(i_{1}+\ldots+i_{p_{1}}, \ldots, i_{p_{m-1}+1}+\ldots+i_{n}\right) \\
& K=\left(j_{1}+\ldots+j_{r_{1}}, \ldots, j_{r_{m-1}+1}+\ldots+j_{s}\right)
\end{aligned}
$$




\section{Definition 5}

$$
\begin{aligned}
P^{J}(t) & =\sum_{I} \varsigma_{I \mid J}(t) S_{I}, \quad \text { where } \\
\varsigma_{I \mid J}(t) & =(-1)^{\ell(I)-\ell(J)} t^{|I|-\sum_{k=1}^{m} i_{p_{k}}} \frac{\prod_{s \in\{1, \ell(J)\} /\left\{r_{k}\right\}}\left(1-t^{\ell(J)-s}\right) \prod_{k=1}^{m}\left(1-t^{\ell(J)-r_{k}+i_{p_{k}}}\right)}{[\ell(J)]_{t} !},
\end{aligned}
$$

Notice that for a given $J$ each polynomial $\varsigma_{J I}(t)$ has the same top degree

$$
\frac{\ell(J)(\ell(J)-1)}{2}+|J|
$$

The first two properties of noncommutative Hall-Littlewood functions follow directly from the definition. The last part of the formula involving products is a direct $t$-generalization of (3) and insures that

$$
P^{J}(1)=M^{J}
$$

The first part of the formula with the power of $t$ ensures that at $t=0$ only the terms corresponding to $I \preceq J$ survive since in that and only that case $K=I$ and $\sum_{k=1}^{m} i_{p_{k}}=|I|$. This ensures that

$$
P^{J}(0)=R^{J}
$$

Further, if one denotes

$$
Q^{J}(t)=[\ell(J)]_{t} ! P^{J}(t)
$$

then the statement is that in the commutative limit it is these function that for hook compositions reduce to classical Hall-Littlewood polynomials.

Consider an example of (4) for $J=1^{2} 2$ 


\section{Example 5}

$$
\begin{aligned}
& Q^{1^{2} 2}(t)=-\varsigma_{1^{4} \mid 1^{2} 2} S_{1}^{4}+\varsigma_{21^{2} \mid 1^{2} 2} S_{21^{2}}+\varsigma_{121 \mid 1^{2} 2} S_{121}+\varsigma_{1^{2} 2 \mid 1^{2}{ }^{2}} S_{1^{2} 2}-\varsigma_{2^{2} \mid 1^{2} 2} S_{2^{2}}-\varsigma_{31 \mid 1^{2} 2} S_{31} \\
& -\varsigma_{13 \mid 1^{2} 2} S_{13}+\varsigma_{4 \mid 1^{2} 2} S_{4} \\
& \varsigma_{1^{4} \mid 1^{2} 2}=\left(1-t^{3-1+1}\right)\left(1-t^{3-2+1}\right)\left(1-t^{3-3+1}\right)=(1-t)\left(1-t^{2}\right)\left(1-t^{3}\right) \\
& K=1^{2} 2 ; \quad i_{p_{1}}=1, i_{p_{2}}=1, i_{p_{3}}=1 ; \quad r_{k}=k ; \quad s=\{\emptyset\} \\
& \varsigma_{21^{2} \mid 1^{2} 2}=\left(1-t^{3-1}\right)\left(1-t^{3-2+2}\right)\left(1-t^{3-3+1}\right)=(1-t)\left(1-t^{2}\right)\left(1-t^{3}\right) \\
& K=2^{2} ; \quad i_{p_{1}}=2, i_{p_{2}}=1 ; \quad r_{1}=2, r_{2}=3 ; \quad s=\{1\} \\
& \varsigma_{121 \mid 1^{2} 2}=\left(1-t^{3-2}\right)\left(1-t^{3-1+1}\right)\left(1-t^{3-3+1}\right)=(1-t)^{2}\left(1-t^{3}\right) \\
& K=13 ; \quad i_{p_{1}}=1, i_{p_{2}}=1 ; \quad r_{1}=1, r_{2}=3 ; \quad s=\{2\} \\
& \varsigma_{1^{2} 2 \mid 1^{2} 2}=\left(1-t^{3-1+1}\right)\left(1-t^{3-2+1}\right)\left(1-t^{3-3+2}\right)=\left(1-t^{2}\right)^{2}\left(1-t^{3}\right) \\
& K=1^{2} 2 ; \quad i_{p_{k}}=i_{k} ; \quad r_{k}=k ; \quad s=\{\emptyset\} \\
& \varsigma_{2^{2} \mid 1^{2} 2}=\left(1-t^{3-1}\right)\left(1-t^{3-2+2}\right)\left(1-t^{3-3+2}\right)=\left(1-t^{2}\right)^{2}\left(1-t^{3}\right) \\
& K=2^{2} ; \quad i_{p_{1}}=2, i_{p_{2}}=2 ; \quad r_{1}=2, r_{2}=3 ; \quad s=\{1\} \\
& \varsigma_{31 \mid 1^{2} 2}=\left(1-t^{3-1}\right)\left(1-t^{3-2}\right)\left(1-t^{3-3+1}\right)=(1-t)^{2}\left(1-t^{2}\right) \\
& K=4 ; \quad i_{p_{1}}=1 ; \quad r_{1}=3 ; \quad s=\{1,2\} \\
& \varsigma_{13 \mid 1^{2} 2}=\left(1-t^{3-2}\right)\left(1-t^{3-1+1}\right)\left(1-t^{3-3+3}\right)=(1-t)\left(1-t^{3}\right)^{2} \\
& K=13 ; \quad i_{p_{1}}=1, i_{p_{2}}=3 ; \quad r_{1}=1, r_{2}=3 ; \quad s=\{2\} \\
& \varsigma_{4 \mid 1^{2} 2}=\left(1-t^{3-1}\right)\left(1-t^{3-2}\right)\left(1-t^{3-3+4}\right)=(1-t)\left(1-t^{2}\right)\left(1-t^{4}\right) \\
& K=4 ; \quad i_{p_{1}}=4 ; \quad r_{1}=3 ; \quad s=\{1,2\} \\
& Q^{1^{2} 2}(t)=-t(1-t)\left(1-t^{2}\right)\left(1-t^{3}\right) S_{1}^{4}+t(1-t)\left(1-t^{2}\right)\left(1-t^{3}\right) S_{21^{2}}+t^{2}(1-t)^{2}\left(1-t^{3}\right) S_{121}+ \\
& +\left(1-t^{2}\right)^{2}\left(1-t^{3}\right) S_{1^{2} 2}-\left(1-t^{2}\right)^{2}\left(1-t^{3}\right) S_{2^{2}}-t^{3}(1-t)^{2}\left(1-t^{2}\right) S_{31}-(1-t)\left(1-t^{3}\right)^{2} S_{13}+ \\
& +(1-t)\left(1-t^{2}\right)\left(1-t^{4}\right) S_{4}
\end{aligned}
$$

I now turn to the multiplication rule for noncommutative Hall-Littlewoods.

\section{Proposition 3}

$$
P^{I}(t) \cdot P^{J}(t)=\sum_{K \preceq I} t^{\operatorname{maj}\left(I_{K}\right)+\left({ }_{2}^{(|K|+1)}\right)}\left\{\left[\begin{array}{c}
\ell(I)+\ell(J) \\
\ell(K)
\end{array}\right]_{t} P^{K \cdot J}(t)+\left[\begin{array}{c}
\ell(I)+\ell(J)-1 \\
\ell(K)
\end{array}\right]_{t} P^{K \triangleright J}(t)\right\},
$$

Proof: The proof is by induction.

It is worth noticing that this formula interpolates between multiplication formulas for noncommutative ribbon Schur functions (at $t=0$ )

$$
R^{I} R^{J}=R^{I \cdot J}+R^{I \triangleright J}
$$


and monomial (at $t=1$ )

$$
M^{I} M^{J}=\sum_{K \preceq I}\left(\begin{array}{c}
\ell(I)+\ell(J) \\
\ell(K)
\end{array}\right) M^{K \cdot J}(t)+\left(\begin{array}{c}
\ell(I)+\ell(J)-1 \\
\ell(K)
\end{array}\right) M^{K \triangleright J}(t)
$$

\section{Expansion of Ribbon Schur in the Hall-Littlewood Basis.}

Finally I would like to address the last property of noncommutative Hall-Littlewood symmetric functions, namely the expansion of ribbon Schur in this basis.

\section{Proposition 4}

$$
R^{I}=\sum_{J} K_{I J}(t) P^{J}(t)
$$

where $K_{I J}(t)$ are polynomials in $t$ with positive coefficients. More precisely,

$$
K_{I J}(t)=\sum_{u=\operatorname{pack}(u), D(u)=I, W C(u)=J} t^{\operatorname{maj}(J)-\sin v(u)}
$$

The proof of this statement requires two steps. First - the expression for the expansion of homogeneous basis in terms of Hall-Littlewood.; second - explicit expression for (7).

\subsection{Relative Decomposition of Two Compositions.}

Consider a decomposition of composition $J$ relative to composition $I$, i.e. a filling of composition $J$ with sub-compositions of weight $\left(i_{1}, i_{2}, \ldots, i_{n}\right)$. Denote by $h_{k}$ the row of $J$ that contains the last cell of $i_{k}$. Rows will be labeled from bottom up starting from zero. Call the sequence (which is actually a partition with some zero parts) of $\left(h_{1}, \ldots, h_{n}\right)$ relative decomposition of $J$ with respect to $I$. (Compare with a related notion in Gelfand et al. (1995)). For instance, consider a decomposition of $J=1323$ with respect to $I=1422$ :

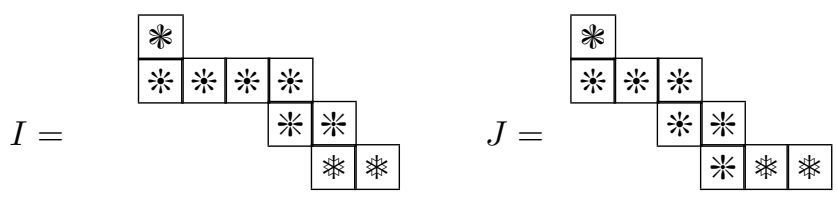

where I have marked all rows of $I$ with different symbols so that one can see where they end up within $J$.

Thus the decomposition of $J=1323$ with respect to $I=1422$ is $h(J, I)=(3,1,0,0)$ Given such a decomposition $h(J, I)$, form a product:

$$
\xi_{I \mid J}=\prod_{k=1}^{\ell(I)-1}\left[\begin{array}{c}
i_{k}+h_{k} \\
i_{k}
\end{array}\right]_{q}
$$


So if $J=1323$ and $I=1422$ as in the example above, then

$$
\xi_{1422 \mid 1323}=\left[\begin{array}{c}
1+3 \\
1
\end{array}\right]_{q}\left[\begin{array}{c}
4+1 \\
4
\end{array}\right]_{q}\left[\begin{array}{c}
2+0 \\
2
\end{array}\right]_{q}
$$

\section{Proposition 5}

$$
\sum_{I \preceq K}(-1)^{\ell(K)-\ell(I)} \prod_{k=1}^{\ell(I)-1}\left[\begin{array}{c}
i_{k}+h_{k} \\
i_{k}
\end{array}\right]_{q}=\sum_{u=\operatorname{pack}(u), D(u)=K, W C(u)=J} q^{\operatorname{sinv}(u)},
$$

Example 6 Let $K=121$ and $J=21^{2}$, then $I_{1}=121, I_{2}=31, I_{3}=13, I_{4}=4$

$$
\begin{aligned}
\xi_{121 \mid 21^{2}}-\xi_{31 \mid 21^{2}}-\xi_{13 \mid 21^{2}}+\xi_{4 \mid 21^{2}} & =\underbrace{\left[\begin{array}{c}
1+2 \\
1
\end{array}\right]_{q}\left[\begin{array}{c}
2+1 \\
2
\end{array}\right]_{q}}_{h\left(21^{2} \mid 121\right)=(2,1)}-\underbrace{\left[\begin{array}{c}
1+2 \\
1
\end{array}\right]_{q}}_{h\left(21^{2} \mid 13\right)=(2,0)}-\underbrace{\left[\begin{array}{c}
3+1 \\
3
\end{array}\right]_{q}}_{h\left(21^{2} \mid 31\right)=(1,0)}+1 \\
& =q^{2}\left(1+q+q^{2}\right)
\end{aligned}
$$

The corresponding packed words are: 2132,3132,3231 and the number of inversions of these words:

$$
\begin{aligned}
& \operatorname{inv}(2132)=2, \operatorname{inv}(3132)=3, \text { inv }(3231)=4 \\
& \text { i.e. the right-hand side is } q^{2}+q^{3}+q^{4}
\end{aligned}
$$

The proof of (9) will be published separately Tishbi and Tevlin (2010).

\subsection{Special Inversion Statistics and the Expansion of Ribbon Schur Functions.}

By induction one can establish the following expansion of noncommutative complete symmetric function in noncommutative Hall-Littlewood basis:

\section{Proposition 6}

$$
S^{I}=\sum_{J} \rho_{I J} H^{J}(t)
$$

where

$$
\rho_{I J}=t^{\operatorname{maj}(J)} \prod_{i=1}^{\ell(I)-1}\left[\begin{array}{c}
i_{k}+h_{k} \\
i_{k}
\end{array}\right]_{\frac{1}{t}}
$$

Note, that $\rho_{I J}$ may be thought of as noncommutative t-supernomial coefficents, compare with Schilling (2002).

Using the definition of ribbon Schur functions (2) and (9) the statement (6) is immediate.

\section{Comments.}

Obviously one would like to know if there is a representation-theoretic and/or geometric interpretation of the above results. In that context it would be interesting to see if there is a noncommutative analog of the plethystic substitution and, therefore, an appropriate analog of modified Hall-Littlewood functions. Also, the question of specialization of noncommutative Hall-Littlewood functions and q-series identities implied by, for instance, multiplication rule, has not been explored. 


\section{Acknowledgements}

I would like to thank Jean-Yves Thibon and Jean-Christophe Novelli for many useful discussions. I am a grateful user of SAGE and I would like to express my thanks to Nicolas Thiery, Florent Hivert, Mike

Hansen, Franco Saliola, Jason Bandlow for helping me to learn the environment.

\section{References}

N. Bergeron and M. Zabrocki. $q$ and $q, t$-analogs of non-commutative symmetric functions. Discrete Math., 298:79-103, 2005.

J. Desarmenien, B. Leclerc, and J.-Y. Thibon. Hall-Littlewood functions and Kostka-Foulkes polynomials in representation theory. In IN REPRESENTATION THEORY, PREPRINT - INSTITUT GASPARD MONGE, pages 94-13, 1994.

I. M. Gelfand, D. Krob, A. Lascoux, B. Leclerc, V. S. Retakh, and Jean-Yves-Thibon. Noncommutative symmetric functions. Advances in Mathematics, 112:218-348, 1995.

F. Hivert. Analogues non-commutatifs et quasi-symétriques des fonctions de Hall-Littlewood et modules de Demazure d'une algèbre quantique dégénérée. C. R. Acad. Sci. Paris, 362:1-6, 1998.

F. Hivert, J.-C. Novelli, L. Tevlin, and J.-Y. Thibon. Permutation statistics related to a class of noncommutative symmetric functions and generalizations of the Genocchi numbers. Selecta Mathematica, 15: 105-119, 2009.

A. N. Kirillov. New combinatorial formula for modified Hall-Littlewood polynomials. Contemp. Math, 254:283-333, 1998.

A. N. Kirillov and N. Y. Reshetikhin. Bethe ansatz and the combinatorics of Young tableaux. J. Sov. Math., 41:925-955, 1988.

A. Lascoux and M. P. Schutzenberger. Sur une conjecture de H.O. Foulkes. C.R. Acad. Sci. Paris, 286A: 323-324, 1978.

I. Macdonald. Symmetric Functions and Hall Polynomials. Oxford Univ. Press, 2 edition, 1995.

J.-C. Novelli, J.-Y. Thibon, and L. Williams. Combinatorial hopf algebras, noncommutative HallLittlewood functions, and permutations=tableaux. Advances in Mathematics, 224(4):1311-1348, 2010.

A. Schilling. q-supernomial coefficients: From riggings to ribbons. In MathPhys Odyssey 2001, pages 437-454. Birkhaeuser, 2002.

L. Tevlin. Noncommutative monomial symmetric functions. In Proc. FPSAC 19 Tianjin, 2007.

N. Tishbi and L. Tevlin. On the number of special inversions of packed words. in preparation, 2010. 
